Electronic Journal of Statistics

Vol. 16 (2022) 1461-1484

ISSN: 1935-7524

https://doi.org/10.1214/21-EJS1975

\title{
Minimax bounds for estimating multivariate Gaussian location mixtures*
}

\author{
Arlene K. H. Kim ${ }^{1}$ and Adityanand Guntuboyina ${ }^{2}$ \\ ${ }^{1}$ Department of Statistics, Korea University, 145 Anam-ro, Seongbuk-gu, Seoul, 02841, \\ South Korea \\ e-mail: arlenent@korea.ac.kr \\ ${ }^{2}$ Department of Statistics, 423 Evans Hall, University of California, Berkeley, CA - 94720, \\ $U S$ \\ e-mail: aditya@stat.berkeley.edu
}

\begin{abstract}
We prove minimax bounds for estimating Gaussian location mixtures on $\mathbb{R}^{d}$ under the squared $L^{2}$ and the squared Hellinger loss functions. Under the squared $L^{2}$ loss, we prove that the minimax optimal rate is upper and lower bounded by a constant multiple of $n^{-1}(\log n)^{d / 2}$. Under the squared Hellinger loss, we consider two subclasses based on the behavior of the tails of the mixing measure. When the mixing measure has a sub-Gaussian tail, the minimax rate under the squared Hellinger loss is bounded from below by $(\log n)^{d} / n$, which implies that the optimal minimax rate is between $(\log n)^{d} / n$ and the upper bound $(\log n)^{d+1} / n$ obtained by [11]. On the other hand, when the mixing measure is only assumed to have a bounded $p^{\text {th }}$ moment for a fixed $p>0$, the minimax rate under the squared Hellinger loss is bounded from below by $n^{-p /(p+d)}(\log n)^{-3 d / 2}$. This rate is minimax optimal up to logarithmic factors.
\end{abstract}

Keywords and phrases: Assouad's lemma, almost parametric rate of convergence, curse of dimensionality, minimax lower bounds, multivariate normal location mixtures.

Received May 2021.

\section{Introduction}

Let $\phi$ be the standard univariate normal density and let $\mathcal{F}_{d}$ denote the class of densities on $\mathbb{R}^{d}$ of the form:

$$
\left(x_{1}, \ldots, x_{d}\right) \mapsto \int \phi\left(x_{1}-u_{1}\right) \phi\left(x_{2}-u_{2}\right) \ldots \phi\left(x_{d}-u_{d}\right) d G\left(u_{1}, \ldots, u_{d}\right),
$$

where $G$ is a probability measure on $\mathbb{R}^{d} . \mathcal{F}_{d}$ is precisely the class of all Gaussian location mixture densities on $\mathbb{R}^{d}$. We study minimax rates in the problem of estimating an unknown density $f^{*} \in \mathcal{F}_{d}$ from i.i.d observations $X_{1}, \ldots, X_{n}$ (throughout the paper, we assume that $n \geq 2$ ). Mixture models are useful in modeling situations involving the presence of subpopulations within an overall

${ }^{*}$ The first author was supported by NRF-2020R1F1A1A01069632. The second author was supported by NSF CAREER Grant DMS-16-54589. 
population and Gaussian location mixtures form the simplest and most commonly used mixture model.

The minimax rate crucially depends on the choice of the loss function. We study two different loss functions in this paper. The first is the squared $L^{2}$ distance:

$$
L^{2}(f, g):=\int(f(\boldsymbol{x})-g(\boldsymbol{x}))^{2} d \boldsymbol{x} .
$$

The minimax risk of estimation over $\mathcal{F}_{d}$ under the $L^{2}$ loss function is

$$
R_{n}\left(\mathcal{F}_{d}, L^{2}\right):=\inf _{\hat{f}_{n}} \sup _{f^{*} \in \mathcal{F}_{d}} \mathbb{E}_{f^{*}} L^{2}\left(\hat{f}_{n}, f^{*}\right) .
$$

In Theorem 2.1, we prove that $R_{n}\left(\mathcal{F}_{d}, L^{2}\right)$ is of the order $n^{-1}(\log n)^{d / 2}$. This result is known for $d=1$. Indeed, when $d=1$, the upper bound follows from the results proved in Ibragimov [5] for estimation of smooth functions (also see Kim [7, Section 3]) and the lower bound was proved by Kim [7]. To the best of our knowledge the result for $d \geq 2$ is novel. It is interesting that the rate $n^{-1}(\log n)^{d / 2}$ has a relatively mild dependence on the dimension $d$ and thus the usual curse of dimensionality is largely avoided for estimating multivariate Gaussian location mixtures under the $L^{2}$ loss function.

The second loss function we investigate is the squared Hellinger distance:

$$
h^{2}(f, g):=\int(\sqrt{f(\boldsymbol{x})}-\sqrt{g(\boldsymbol{x})})^{2} d \boldsymbol{x} .
$$

Unlike the squared $L^{2}$ distance, the squared Hellinger distance does not depend on the choice of dominating measure and can thus be viewed as a discrepancy measure between the underlying probability measures. Compared to the squared $L^{2}$ distance, the squared Hellinger distance is much more sensitive to estimation errors in the small-density regions. Further, estimation accuracy under the squared Hellinger distance is connected to the squared error accuracy of certain empirical Bayes estimates of normal means [6, 11].

In order to obtain meaningful rates under the squared Hellinger distance, it is necessary to impose additional conditions on the probability measure $G$ underlying the density (1.1). The most common assumption in the literature is to assume that $G$ is discrete with a known upper bound on the number of atoms. The Hellinger accuracy (as well as accuracy in the total variation distance) of estimating discrete Gaussian location mixtures has been investigated, for example, in $[1,4,8,12,14]$. In particular, it was proved by Doss, Wu, Yang and Zhou [1] (and Wu and Yang [14] for $d=1$ ) that the minimax rate is $n^{-1}$ when the dimension $d$ and the number of atoms of $G$ are bounded from above by constants.

In contrast to the discrete mixture situation, minimax rates using squared Hellinger distance under broader assumptions on $G$ are not fully understood. Given a subclass $\mathcal{G}$ of probability measures on $\mathbb{R}^{d}$, let $\mathcal{F}_{\mathcal{G}}$ denote the class of all densities of the form (1.1) where $G$ is constrained to be in $\mathcal{G}$. We shall denote 
the minimax risk over $\mathcal{F}_{\mathcal{G}}$ in the squared Hellinger distance by

$$
R_{n}\left(\mathcal{F}_{\mathcal{G}}, h^{2}\right):=\inf _{\hat{f}_{n}} \sup _{f^{*} \in \mathcal{F}_{\mathcal{G}}} \mathbb{E}_{f^{*}} h^{2}\left(\hat{f}_{n}, f^{*}\right) .
$$

We study $R_{n}\left(\mathcal{F}_{\mathcal{G}}, h^{2}\right)$ for the following two natural subclasses $\mathcal{G}$ :

1. $\mathcal{G}=\mathcal{G}_{1}(\Gamma)$ : the class of all probability measures $G$ satisfying $G\{\boldsymbol{u}:\|\boldsymbol{u}\|>$ $t\} \leq C \exp \left(-t^{2} / 2 \Gamma\right)$ for some constant $C$ and all $t>0$ and a constant $\Gamma$. Every probability measure in $\mathcal{G}_{1}(\Gamma)$ has sub-Gaussian tails.

2. $\mathcal{G}=\mathcal{G}_{2}(p, K)$ : the class of all probability measures $G$ satisfying

$$
\left(\int\|\boldsymbol{u}\|^{p} d G(\boldsymbol{u})\right)^{1 / p} \leq K
$$

for a fixed $p>0$ and constant $K>0$.

The problem of estimation of densities belonging to the classes $\mathcal{F}_{\mathcal{G}_{1}(\Gamma)}$ and $\mathcal{F}_{\mathcal{G}_{2}(p, K)}$ has been studied in $[2,15,7]$ for $d=1$ and in [11] for $d \geq 1$. Extending the results of Zhang [15] to $d \geq 1$, Saha and Guntuboyina [11] analyzed the performance of the nonparametric maximum likelihood estimator over $\mathcal{F}_{d}$ leading to the following upper bounds on $R_{n}\left(\mathcal{F}_{\mathcal{G}_{1}(\Gamma)}, h^{2}\right)$ and $R_{n}\left(\mathcal{F}_{\mathcal{G}_{2}(p, K)}, h^{2}\right)$ :

$$
R_{n}\left(\mathcal{F}_{\mathcal{G}_{1}(\Gamma)}, h^{2}\right) \leq C_{d, \Gamma} \frac{(\log n)^{d+1}}{n},
$$

and

$$
R_{n}\left(\mathcal{F}_{\mathcal{G}_{2}(p, K)}, h^{2}\right) \leq C_{d, K, p} n^{-\frac{p}{p+d}}(\log n)^{\frac{2 d+2 p+d p}{2 p+2 d}} .
$$

To the best of our knowledge, the corresponding lower bounds do not currently exist in the literature (except for the case of $R_{n}\left(\mathcal{F}_{\mathcal{G}_{1}(\Gamma)}, h^{2}\right)$ for $d=1$ ) and we establish these in this paper. Specifically, we prove that

$$
R_{n}\left(\mathcal{F}_{\mathcal{G}_{1}(\Gamma)}, h^{2}\right) \geq c_{d, \Gamma} \frac{(\log n)^{d}}{n}
$$

and

$$
R_{n}\left(\mathcal{F}_{\mathcal{G}_{2}(p, K)}, h^{2}\right) \geq c_{d, K, p} n^{-\frac{p}{p+d}}(\log n)^{-\frac{3 d}{2}}
$$

in Theorem 2.2 and Theorem 2.3 respectively.

(1.6) implies that there is a logarithmic price to be paid for dimensionality under the sub-Gaussianity assumption. (1.7) implies that the rate of convergence becomes much slower (than the parametric rate) if we only assume boundedness of the $p^{\text {th }}$ moment of $G$ for a fixed $p>0$. It is usually believed that Gaussian location mixtures are arbitrarily smooth leading to nearly parametric rates of estimation. While this is true for the $L^{2}$ loss function, our results reveal that the story is more complicated for the squared Hellinger loss function. Specifically, inequality (1.7) shows that, under the squared Hellinger distance, the rates can be arbitrarily slow if the mixing measure is allowed to have heavy tails. This fact (which can also be understood by considering $n$-component mixture densities 
with well-separated centers) does not seem to have been emphasized previously in the literature even for $d=1$. Furthermore, for each fixed $p$, the rate becomes exponentially slow in $d$ revealing the usual curse of dimensionality. Note that our lower bounds also imply that the upper bounds (1.4) and (1.5) cannot be substantially improved.

The rest of the paper is organized as follows. Our main results are all stated in the next section. Theorem 2.1 proves the minimax rate of $(\log n)^{d / 2} / n$ for $\mathcal{F}_{d}$ under the $L^{2}$ loss. Theorem 2.2 and Theorem 2.3 deal with the squared Hellinger loss function: Theorem 2.2 proves the minimax lower bound of $(\log n)^{d} / n$ under the subgaussianity assumption on the mixing measure and Theorem 2.3 proves the $n^{-p /(p+d)}(\log n)^{-3 d / 2}$ lower bound under the bounded $p^{t h}$ moment assumption on the mixing measure. The proofs of these results are given in Section 3. We also recall in this section (see Subsection 3.1) some basic facts about Fourier transforms, Hermite polynomials and Assouad's lemma that are used in our proofs.

\section{Main results}

We state all our main results in this section. Our first result shows that the minimax risk $R_{n}\left(\mathcal{F}_{d}, L^{2}\right)$ is of the order $n^{-1}(\log n)^{d / 2}$.

Theorem 2.1. Let $d \in \mathbb{N}$. There exist positive constants $c_{d}$ and $C_{d}$ depending only on d such that

$$
c_{d} \frac{(\log n)^{d / 2}}{n} \leq R_{n}\left(\mathcal{F}_{d}, L^{2}\right) \leq C_{d} \frac{(\log n)^{d / 2}}{n} .
$$

The proof of the upper bound on $R_{n}\left(\mathcal{F}_{d}, L^{2}\right)$ in $(2.1)$ is based on an extension of the ideas of [5] to $d \geq 1$ (a simple exposition of these ideas can be found in Kim [7, Theorem 4.1]). It involves considering the estimator

$$
\hat{f}_{n}(\boldsymbol{x}):=\frac{1}{n h^{d}} \sum_{i=1}^{n} \mathbb{K}\left(\frac{X_{i}-\boldsymbol{x}}{h}\right)
$$

where $\mathbb{K}(\boldsymbol{y}):=K\left(y_{1}\right) \ldots K\left(y_{d}\right)$ with $K(y):=(\sin y) /(\pi y)$ and the bandwidth $h$ is taken to be $h:=(2 \log n)^{-1 / 2}$. Controlling the variance of $\hat{f}_{n}(\boldsymbol{x})$ is straightforward while bounding the bias is non-trivial and we do this via Fourier analysis.

The proof of the lower bound on $R_{n}\left(\mathcal{F}_{d}, L^{2}\right)$ in (2.1) is based on an extension of the ideas of Kim [7, Proof of Theorem 1.1]. It involves applying Assouad's Lemma (recalled in Lemma 3.1) to a carefully chosen subset of $\mathcal{F}_{d}$ whose elements are indexed by a hypercube. This subset of $\mathcal{F}_{d}$ is constructed by taking mixing measures that are additive perturbations of a Gaussian mixing measure. The additive perturbations are created using Hermite polynomials.

Our next result proves a lower bound of order $(\log n)^{d} / n$ for $R_{n}\left(\mathcal{F}_{\mathcal{G}_{1}(\Gamma)}, h^{2}\right)$. A comparison with the upper bound (1.4) of Saha and Guntuboyina [11] reveals that this lower bound is possibly off by at most a factor of $\log n$ and is thus minimax rate optimal up to the single $\log n$ multiplicative factor. 
Theorem 2.2. Let $d \in \mathbb{N}$ and $\Gamma>0$. There exists a positive constant $c_{d, \Gamma}$ depending only on $d$ and $\Gamma$ such that

$$
R_{n}\left(\mathcal{F}_{\mathcal{G}_{1}(\Gamma)}, h^{2}\right) \geq c_{d, \Gamma} \frac{(\log n)^{d}}{n} .
$$

For a recent related result, please see [9] as well. The proof of Theorem 2.2 is based on an extension of the ideas of Kim [7, Proof of Theorem 1.3]. Assouad's lemma is applied to a subset of $\mathcal{F}_{\mathcal{G}_{1}(\Gamma)}$ which is constructed by taking mixing measures that are additive perturbations of a Gaussian mixing measure. The perturbations are different from those used in the proof of Theorem 2.1 although they are also based on Hermite polynomials. It is not easy to directly work with the squared Hellinger loss function while dealing with mixture densities so we crucially use the fact that the squared Hellinger loss is bounded from below by a constant multiple of the chi-squared divergence for the constructed subset of $\mathcal{F}_{d}$.

Our final result proves a lower bound of the order $n^{-p /(p+d)}$ (up to a logarithmic factor) for $R_{n}\left(\mathcal{F}_{\mathcal{G}_{2}(p, K)}, h^{2}\right)$. As we mentioned previously, this result reveals that rates strictly slower than $n^{-1}$ are possible for estimating Gaussian location mixtures and that the rate can also be affected by the usual curse of dimensionality. A comparison with the upper bound (1.5) of Saha and Guntuboyina [11] reveals that this lower bound is optimal up to logarithmic factors possibly depending on $d$.

Theorem 2.3. Let $d \in \mathbb{N}$ and $K>0$. For every $p>0$, there exists a positive constant $c_{d, K, p}$ depending only on $d, K$ and $p$ such that

$$
R_{n}\left(\mathcal{F}_{\mathcal{G}_{2}(p, K)}, h^{2}\right) \geq c_{d, K, p} n^{-\frac{p}{p+d}}(\log n)^{-\frac{3 d}{2}} .
$$

For the proof of Theorem 2.3, we first construct a normal mixture density whose mixing measure is a discrete distribution that is supported on a $d$-dimensional product set (lattice) and has a Pareto type tail behavior. We then construct a hypercube of normal mixture densities by perturbing this support set. For each point in the support set, we use either the original point or a nearby point whose distance to the non-perturbed point is determined by the probability value at the original support point of the mixing distribution. We finally apply Assouad's lemma to the constructed hypercube of densities in $\mathcal{F}_{\mathcal{G}_{2}(p, K)}$

The proofs of our results are given in the next section.

\section{Proofs}

\subsection{Preliminaries}

We shall recall here some standard facts about the Fourier transform and Hermite polynomials that we shall use in our main proofs. 
We use the notation $\mathcal{T}$ for the Fourier transform. It is defined as

$$
\mathcal{T}(f)(\boldsymbol{t})=(2 \pi)^{-d / 2} \int e^{-i t_{1} x_{1}-i t_{2} x_{2}-\cdots-i t_{d} x_{d}} f(\boldsymbol{x}) d \boldsymbol{x}
$$

for functions $f \in L_{1}\left(\mathbb{R}^{d}\right)$. The Fourier inversion theorem gives

$$
f(x)=(2 \pi)^{-d / 2} \int e^{i x_{1} t_{1}+\cdots+i x_{d} t_{d}} \mathcal{T}(f)(\boldsymbol{t}) d \boldsymbol{t} .
$$

Plancherel's theorem states that

$$
\int|f(\boldsymbol{x})|^{2} d \boldsymbol{x}=\int|\mathcal{T}(f)(\boldsymbol{t})|^{2} d \boldsymbol{t}
$$

for functions $f \in L_{1}\left(\mathbb{R}^{d}\right) \cap L_{2}\left(\mathbb{R}^{d}\right)$. The convolution-product property of the Fourier transform states that

$$
\mathcal{T}(h)(\boldsymbol{t})=\mathcal{T}(f)(\boldsymbol{t}) \mathcal{T}(g)(\boldsymbol{t}) \text { for all } \boldsymbol{t} \text { where } h(\boldsymbol{x}):=\int f(\boldsymbol{x}-\boldsymbol{u}) g(\boldsymbol{u}) d \boldsymbol{u} .
$$

Our upper bound on $R_{n}\left(\mathcal{F}_{d}, L^{2}\right)$ is based on the sinc kernel $\mathbb{K}$ with $\mathbb{K}(\boldsymbol{y}):=$ $K\left(y_{1}\right) \ldots K\left(y_{d}\right)$ where

$$
K(y):=\frac{\sin y}{\pi y} .
$$

It is well-known that

$$
\begin{aligned}
K(y) & =K(-y), \quad \mathcal{T}(K)(t)=\frac{1}{\sqrt{2 \pi}} \mathbb{1}_{\{|t| \leq 1\}}, \quad \text { and } \\
\mathcal{T}\left(K^{2}\right)(t) & =\frac{1}{\pi \sqrt{2 \pi}}\left(1-\frac{|t|}{2}\right)_{+}
\end{aligned}
$$

where $x_{+}:=\max (x, 0)$.

Our lower bound constructions involve Hermite polynomials. These are defined for $d=1$ as

$$
H_{j}(x):=(-1)^{j} e^{x^{2} / 2} \frac{d^{j}}{d x^{j}} e^{-x^{2} / 2}=\frac{(-1)^{j}}{\phi(x)} \frac{d^{j} \phi(x)}{d x^{j}} .
$$

Note that $H_{j}(\cdot)$ is an odd function when $j$ is odd (and even when $j$ is even). The Hermite polynomials are orthogonal with respect to the weight function $\phi(x):=(2 \pi)^{-1 / 2} \exp \left(-x^{2} / 2\right)$. Specifically for all $j \geq 0$ and $k \geq 0$, we have

$$
\int \phi(x) H_{j}(x) H_{k}(x) d x= \begin{cases}0 & \text { for } j \neq k \\ j ! & \text { for } j=k\end{cases}
$$

We shall use the bound

$$
\left|H_{j}(x)\right| \leq \kappa \sqrt{j !} \exp \left(x^{2} / 4\right)
$$


for a constant $\kappa \sim 1.086<2^{1 / 4}$ (see e.g. Equation 8.954 of [3]).

For $d \geq 1$, we take

$$
H_{j}(\boldsymbol{x}):=\frac{(-1)^{|\boldsymbol{j}|}}{\boldsymbol{\phi}(\boldsymbol{x})}\left(\frac{\partial}{\partial \boldsymbol{x}}\right)^{j} \phi(\boldsymbol{x})=H_{j_{1}}\left(x_{1}\right) \ldots H_{j_{d}}\left(x_{d}\right)
$$

where $\phi(\boldsymbol{x})=\phi\left(x_{1}\right) \ldots \phi\left(x_{d}\right),(\partial / \partial \boldsymbol{x})^{\boldsymbol{j}}:=\partial^{j_{1}+\ldots+j_{d}} / \partial x_{1}^{j_{1}} \ldots \partial x_{d}^{j_{d}}$ and $|\boldsymbol{j}|=$ $j_{1}+\ldots+j_{d}$.

We next recall Assouad's lemma (see e.g., [13, Lemma 24.3]) which will be our main tool for proving minimax lower bounds.

Lemma 3.1 (Assouad). Let $d^{2}$ be either (1.2) or (1.3) and define the minimax risk

$$
R_{n}\left(\mathcal{F}_{d}, d^{2}\right):=\inf _{\hat{f}_{n}} \sup _{f^{*} \in \mathcal{F}_{d}} \mathbb{E}_{f^{*}} d^{2}\left(\hat{f}_{n}, f^{*}\right) .
$$

For some $N \geq 1$, let $\left\{f_{\boldsymbol{\tau}}, \boldsymbol{\tau} \in\{0,1\}^{N}\right\}$ be a subset of $\mathcal{F}_{d}$. Then

$$
R_{n}\left(\mathcal{F}_{d}, d^{2}\right) \geq \frac{N}{8} \min _{\boldsymbol{\tau} \neq \boldsymbol{\tau}^{\prime}} \frac{d^{2}\left(f_{\boldsymbol{\tau}}, f_{\boldsymbol{\tau}^{\prime}}\right)}{\Upsilon\left(\boldsymbol{\tau}, \boldsymbol{\tau}^{\prime}\right)} \min _{\Upsilon\left(\boldsymbol{\tau}, \boldsymbol{\tau}^{\prime}\right)=1}\left(1-\sqrt{\frac{n}{2} \chi^{2}\left(f_{\boldsymbol{\tau}} \| f_{\boldsymbol{\tau}^{\prime}}\right)}\right)
$$

where $\Upsilon\left(\boldsymbol{\tau}, \boldsymbol{\tau}^{\prime}\right):=\sum_{j=1}^{N} \mathbb{1}_{\left\{\tau_{j} \neq \tau_{j}^{\prime}\right\}}$ is the Hamming distance between $\boldsymbol{\tau}$ and $\boldsymbol{\tau}^{\prime}$ and $\chi^{2}(f \| g):=\int(f-g)^{2} / g$ is the $\chi^{2}$-divergence between densities $f$ and $g$.

\subsection{Proof of Theorem 2.1}

We break this proof into two parts: the upper bound on $R_{n}\left(\mathcal{F}_{d}, L^{2}\right)$ and the lower bound on $R_{n}\left(\mathcal{F}_{d}, L^{2}\right)$. Let us first prove the upper bound.

We consider the kernel estimator (2.2) with bandwidth $h=(2 \log n)^{-1 / 2}$. We need to control its variance and squared bias. We first bound the variance as

$$
\begin{aligned}
\mathbb{E}_{f} \int\left(\hat{f}_{n}(\boldsymbol{y})-\mathbb{E} \hat{f}_{n}(\boldsymbol{y})\right)^{2} d \boldsymbol{y} & =\frac{1}{n h^{2 d}} \int \operatorname{var}_{f}\left(\mathbb{K}\left(\frac{X_{1}-y}{h}\right)\right) d \boldsymbol{y} \\
& \leq \frac{1}{n h^{2 d}} \int \mathbb{E}_{f} \mathbb{K}^{2}\left(\frac{X_{1}-y}{h}\right) d \boldsymbol{y} \\
& =\frac{1}{n h^{2 d}} \iint \prod_{i=1}^{d} K^{2}\left(\frac{x_{i}-y_{i}}{h}\right) f(\boldsymbol{x}) d \boldsymbol{x} d \boldsymbol{y} \\
& \leq \frac{(2 \pi)^{-d / 2}}{n h^{d}}\left(\int K^{2}(z) d z\right)^{d} \leq \frac{1}{n h^{d}},
\end{aligned}
$$

where the penultimate inequality uses

$$
f(\boldsymbol{x})=\int \phi\left(x_{1}-u_{1}\right) \phi\left(x_{2}-u_{2}\right) \ldots \phi\left(x_{d}-u_{d}\right) d G\left(u_{1}, \ldots, u_{d}\right) \leq(2 \pi)^{-d / 2}
$$

and the last inequality uses $\int K^{2}(z) d x=\int|\mathcal{T}(K)(z)|^{2} d z=1 / \pi$. 
For the bias, note that $\boldsymbol{y} \mapsto \mathbb{E}_{f^{*}} \hat{f}_{n}(\boldsymbol{y})$ has Fourier transform

$$
\boldsymbol{t} \mapsto(2 \pi)^{d / 2} \mathcal{T}(f)(\boldsymbol{t}) \prod_{i=1}^{d} \mathcal{T}(K)\left(h t_{i}\right) .
$$

This is because

$$
\mathbb{E}_{f} \hat{f}_{n}(\boldsymbol{y})=\frac{1}{h^{d}} \int \prod_{i=1}^{d} K\left(\frac{x_{i}-y_{i}}{h}\right) f(\boldsymbol{x}) d \boldsymbol{x}
$$

and

$$
\begin{aligned}
\mathcal{T}\left(\mathbb{E}_{f} \hat{f}_{n}\right)(\boldsymbol{t}) & =\frac{1}{(2 \pi)^{d} h^{d}} \int e^{-i \boldsymbol{t}^{T} \boldsymbol{y}}\left[\prod_{i=1}^{d} K\left(\frac{x_{i}-y_{i}}{h}\right) f(\boldsymbol{x}) d \boldsymbol{x}\right] d \boldsymbol{y} \\
= & \frac{1}{(2 \pi)^{d / 2} h^{d}} \int \prod_{i=1}^{d}\left(\int e^{-i t_{i} y_{i}} K\left(-\frac{x_{i}-y_{i}}{h}\right) d y_{i}\right) f(\boldsymbol{x}) d \boldsymbol{x} \\
= & \frac{1}{(2 \pi)^{d / 2} h^{d}} \int \prod_{i=1}^{d}\left(\int e^{-i t_{i}\left(y_{i}-x_{i}\right)} K\left(\frac{y_{i}-x_{i}}{h}\right) d y_{i}\right) e^{-i \boldsymbol{t}^{T} \boldsymbol{x}} f(\boldsymbol{x}) d \boldsymbol{x} \\
= & \prod_{i=1}^{d}\left(\int \frac{1}{\sqrt{2 \pi}} e^{-i\left(h t_{i}\right) z_{i}} K\left(z_{i}\right) d z_{i}\right) \int e^{-i \boldsymbol{t}^{T} \boldsymbol{x}} f(\boldsymbol{x}) d \boldsymbol{x} \\
= & \prod_{i=1}^{d} \mathcal{T}(K)\left(h t_{i}\right)(2 \pi)^{d / 2} \mathcal{T}(f)(\boldsymbol{t}) .
\end{aligned}
$$

Thus by Plancherel theorem (and (3.1))

$$
\begin{aligned}
\operatorname{bias}^{2} & =\int\left(\mathbb{E}_{f} \hat{f}_{n}(\boldsymbol{y})-f(\boldsymbol{y})\right)^{2}=\int\left|\mathcal{T}\left(\mathbb{E}_{f} \hat{f}_{n}\right)(\boldsymbol{t})-\mathcal{T}(f)(\boldsymbol{t})\right|^{2} d \boldsymbol{t} \\
& =\int|\mathcal{T}(f)(\boldsymbol{t})|^{2}\left|(2 \pi)^{d / 2} \prod_{i=1}^{d} \mathcal{T}(K)\left(h t_{i}\right)-1\right|^{2} d \boldsymbol{t} \\
& =\int|\mathcal{T}(f)(\boldsymbol{t})|^{2}\left|\mathbb{1}_{\left\{\left|t_{1}\right| \leq 1 / h, \ldots,\left|t_{d}\right| \leq 1 / h\right\}}-1\right|^{2} d \boldsymbol{t} \\
& \leq \sum_{i=1}^{d} \int_{\left|t_{i}\right| \geq 1 / h}|\mathcal{T}(f)(\boldsymbol{t})|^{2} d \boldsymbol{t},
\end{aligned}
$$

where the last inequality follows since

$$
\left|\mathbb{1}_{\left\{\left|t_{1}\right| \leq 1 / h, \ldots,\left|t_{d}\right| \leq 1 / h\right\}}-1\right|^{2} \leq \sum_{i=1}^{d} \mathbb{1}_{\left\{\left|t_{i}\right| \geq 1 / h\right\}} .
$$

Consider the first term in the above sum. We split the integral over $\left|t_{1}\right| \geq 1 / h$ into over $t_{1} \leq-1 / h$ and $t_{1} \geq 1 / h$ and observe

$$
\int_{t_{1} \leq-1 / h}|\mathcal{T}(f)(\boldsymbol{t})|^{2} d \boldsymbol{t} \leq e^{-w / h} \int e^{-w t_{1}}|\mathcal{T}(f)(\boldsymbol{t})|^{2} d \boldsymbol{t}
$$




$$
=e^{-w / h} \lim _{M \rightarrow \infty} \int e^{-w t_{1}}|\mathcal{T}(f)(\boldsymbol{t})|^{2} \prod_{i=1}^{d}\left[\left(1-\frac{\left|t_{i}\right|}{M}\right)_{+}\right] d \boldsymbol{t} .
$$

for every $w \geq 0$. The last integral above can be written as

$$
\int e^{-w t_{1}}|\mathcal{T}(f)(\boldsymbol{t})|^{2} \prod_{i=1}^{d}\left[\left(1-\frac{\left|t_{i}\right|}{M}\right)_{+}\right] d \boldsymbol{t}=\int \mathcal{T}(f)(\boldsymbol{t}) e^{-w t_{1}} \overline{\mathcal{T}(\vartheta)(\boldsymbol{t})} d \boldsymbol{t}
$$

where

$$
\mathcal{T}(\vartheta)(\boldsymbol{t})=\mathcal{T}(f)(\boldsymbol{t}) \prod_{i=1}^{d}\left[\left(1-\frac{\left|t_{i}\right|}{M}\right)_{+}\right],
$$

which is the Fourier transform of the following nonnegative function

$$
\vartheta(\boldsymbol{y})=\left(\frac{M \pi^{2}}{\sqrt{2 \pi}}\right)^{d} \int f(\boldsymbol{u}) \prod_{i=1}^{d} K^{2}\left(\frac{M}{2}\left(y_{i}-u_{i}\right)\right) d \boldsymbol{u}
$$

The function $K^{2}$ shows up in the right hand side above essentially because of the third fact in (3.1).

Continuing from (3.8), we get

$$
\begin{aligned}
\int e^{-w t_{1}}|\mathcal{T}(f)(\boldsymbol{t})|^{2} & \prod_{i=1}^{d}\left[\left(1-\frac{\left|t_{i}\right|}{M}\right)_{+}\right] d \boldsymbol{t}=\int \mathcal{T}(f)(\boldsymbol{t}) e^{-w t_{1}} \overline{\mathcal{T}(\vartheta)(\boldsymbol{t})} d \boldsymbol{t} \\
& =\int \mathcal{T}(f)(\boldsymbol{t}) e^{-w t_{1}} \mathcal{T}(\vartheta)(-\boldsymbol{t}) d \boldsymbol{t} \\
& =\int \mathcal{T}(f)(-\boldsymbol{t}) e^{w t_{1}} \mathcal{T}(\vartheta)(\boldsymbol{t}) d \boldsymbol{t} \\
& =\int(2 \pi)^{-d / 2}\left(\int e^{\sum_{j} i t_{j} y_{j}} f(\boldsymbol{y}) d \boldsymbol{y}\right) e^{w t_{1}} \mathcal{T}(\vartheta)(\boldsymbol{t}) d \boldsymbol{t} \\
& =\int(2 \pi)^{-d / 2}\left(\int e^{i \sum_{j} t_{j} y_{j}} e^{w t_{1}} \mathcal{T}(\vartheta)(\boldsymbol{t}) d \boldsymbol{t}\right) f(\boldsymbol{y}) d \boldsymbol{y} \\
& =\int(2 \pi)^{-d / 2}\left(\int e^{i t_{1}\left(y_{1}-i w\right)+i \sum_{j \geq 2} t_{j} y_{j}} \mathcal{T}(\vartheta)(\boldsymbol{t}) d \boldsymbol{t}\right) f(\boldsymbol{y}) d \boldsymbol{y} \\
& =\int \vartheta\left(y_{1}-i w, y_{2}, \ldots, y_{d}\right) f(\boldsymbol{y}) d \boldsymbol{y} \\
& =\int f\left(y_{1}+i w, y_{2}, \ldots, y_{d}\right) \vartheta(\boldsymbol{y}) d \boldsymbol{y} \\
& \leq \sup _{\boldsymbol{y}}\left|f\left(y_{1}+i w, y_{2}, \ldots, y_{d}\right)\right| \int \vartheta(\boldsymbol{y}) d \boldsymbol{y}
\end{aligned}
$$

Note now that

$$
\sup _{\boldsymbol{y}}\left|f\left(y_{1}+i w, y_{2}, \ldots, y_{d}\right)\right| \leq
$$




$$
\begin{aligned}
& \frac{1}{\sqrt{2 \pi}} \sup _{\boldsymbol{y}} \int\left|\exp \left(-\frac{\left(y_{1}+i w-u_{1}\right)^{2}}{2}\right)\right| \prod_{i=2}^{d} \phi\left(y_{i}-u_{i}\right) d G(\boldsymbol{u}) \\
& \leq \frac{1}{\sqrt{2 \pi}} \exp \left(\frac{w^{2}}{2}\right) \int(2 \pi)^{-\frac{(d-1)}{2}} d G(\boldsymbol{u}) \leq(2 \pi)^{-d / 2} \exp \left(\frac{w^{2}}{2}\right),
\end{aligned}
$$

Note also that

$$
\int \vartheta(\boldsymbol{y}) d \boldsymbol{y}=(2 \pi)^{d / 2} \mathcal{T}(\vartheta)(0)=(2 \pi)^{d / 2} \mathcal{T}(f)(0)=1 .
$$

We have thus proved

$$
\begin{aligned}
\int_{t_{1} \leq-1 / h}|\mathcal{T}(f)(\boldsymbol{t})|^{2} d \boldsymbol{t} & \leq e^{-w / h} \int e^{-w t_{1}}|\mathcal{T}(f)(\boldsymbol{t})|^{2} \prod_{i=1}^{d}\left[\left(1-\frac{\left|t_{i}\right|}{M}\right)_{+}\right] d \boldsymbol{t} \\
& \leq(2 \pi)^{-d / 2} \exp \left(-\frac{w}{h}+\frac{w^{2}}{2}\right) .
\end{aligned}
$$

By an entirely analogous argument, it follows that the same bound also holds for the integral over $t_{1} \geq 1 / h$. This gives

$$
\int_{\left|t_{1}\right| \geq 1 / h}|\mathcal{T}(f)(\boldsymbol{t})|^{2} d \boldsymbol{t} \leq 2(2 \pi)^{-d / 2} \exp \left(-\frac{w}{h}+\frac{w^{2}}{2}\right) .
$$

We can analogously prove the same bound for every $i=1, \ldots, d$ leading to

$$
\operatorname{bias}^{2} \leq \sum_{i=1}^{d} \int_{\left|t_{i}\right| \geq 1 / h}|\mathcal{T}(f)(\boldsymbol{t})|^{2} d \boldsymbol{t} \leq 2 d(2 \pi)^{-d / 2} \exp \left(-\frac{w}{h}+\frac{w^{2}}{2}\right)
$$

for every $w \geq 0$. The choice $w=1 / h$ now leads to

$$
\operatorname{bias}^{2} \leq 2 d(2 \pi)^{-d / 2} \exp \left(\frac{-1}{2 h^{2}}\right) .
$$

Combining this with (3.7), we get

$$
R_{n}\left(\mathcal{F}_{d}, L^{2}\right) \leq \inf _{h>0}\left(\frac{1}{n h^{d}}+2 d(2 \pi)^{-d / 2} \exp \left(\frac{-1}{2 h^{2}}\right)\right) .
$$

The choice $h:=(2 \log n)^{-1 / 2}$ then clearly leads to $R_{n}\left(\mathcal{F}_{d}, L^{2}\right) \leq C_{d}(\log n)^{d / 2} / n$ which proves the upper bound.

We now prove the lower bound on $R_{n}\left(\mathcal{F}_{d}, L^{2}\right)$. The idea is to construct a subset of $\mathcal{F}_{d}$ indexed by a hypercube $\{0,1\}^{N}$ for some $N$ and then use Assouad's lemma (Lemma 3.1). Our construction is a natural extension to $d \geq 1$ of the one-dimensional construction in [7] and is described below. Let $m$ be the largest integer such that

$$
\frac{m^{5 d / 4} 8^{d} 3^{d m}}{\sqrt{n}} \leq 1
$$


We can assume without loss of generality that $n$ is large enough so that $m$ defined as above satisfies $m \geq 3$. It is also easy to check (because $m^{5 d / 4} 8^{d} 3^{d m} \geq e^{m}$ ) that the above condition for $m$ implies that

$$
m \leq \frac{1}{2} \log n .
$$

Below we denote by $\phi_{\sigma^{2}}(\cdot)$, the univariate normal density with mean zero and variance $\sigma^{2}$ i.e., $\phi_{\sigma^{2}}(x):=(\sqrt{2 \pi} \sigma)^{-1} \exp \left(-x^{2} /\left(2 \sigma^{2}\right)\right)$. We construct densities (for the application of Assouad's lemma) via perturbations of the density:

$f_{0}(\boldsymbol{x}):=\int \phi\left(x_{1}-u_{1}\right) \ldots \phi\left(x_{d}-u_{d}\right) \gamma(\boldsymbol{u}) d \boldsymbol{u}$ where $\gamma(\boldsymbol{u}):=\phi_{m}\left(u_{1}\right) \ldots \phi_{m}\left(u_{d}\right)$.

Note also that

$$
f_{0}(\boldsymbol{x})=\phi_{1+m}\left(x_{1}\right) \ldots \phi_{1+m}\left(x_{d}\right)
$$

Now let

$$
\mathcal{J}:=\{1,3, \ldots, 2 m-1\}^{d}
$$

and note that cardinality of $\mathcal{J}$ is $|\mathcal{J}|=m^{d}$. We shall apply Assouad's lemma (Lemma 3.1) with $N:=m^{d}$ and we index binary vectors in $\{0,1\}^{N}$ by elements $\boldsymbol{j}:=\left(j_{1}, \ldots, j_{d}\right)$ of $\mathcal{J}$. For $\boldsymbol{\alpha}=\left(\alpha_{\boldsymbol{j}}, \boldsymbol{j} \in \mathcal{J}\right)$, let

$$
f_{\boldsymbol{\alpha}}(\boldsymbol{x})=\int \phi\left(x_{1}-u_{1}\right) \ldots \phi\left(x_{d}-u_{d}\right)\left[\gamma(\boldsymbol{u})+\epsilon \sum_{\boldsymbol{j} \in \mathcal{J}} \alpha_{\boldsymbol{j}} \gamma_{\boldsymbol{j}}(\boldsymbol{u})\right] d \boldsymbol{u}
$$

where $\epsilon$ is given by

$$
\epsilon=c n^{-1 / 2} m^{-d / 4}
$$

for a constant $c \in(0,1)$ that will be determined later and

$$
\begin{aligned}
\gamma_{\boldsymbol{j}}(\boldsymbol{u}) & :=\gamma_{j_{1}}\left(u_{1}\right) \ldots \gamma_{j_{d}}\left(u_{d}\right) \\
& \text { with } \gamma_{j_{i}}\left(u_{i}\right):=2^{1 / 2}(2 \pi)^{3 / 4} \sqrt{\frac{3^{j_{i}}}{j_{i} !}} \phi\left(u_{i}\right) H_{j_{i}}\left(\frac{2}{\sqrt{3}} u_{i}\right) .
\end{aligned}
$$

Here $H_{j_{i}}(\cdot)$ denotes the Hermite polynomial (see (3.2)). Let us first argue that $f_{\alpha} \in \mathcal{F}_{d}$. To see this, it is enough to show that

$$
\boldsymbol{u} \mapsto \gamma(\boldsymbol{u})+\epsilon \sum_{\boldsymbol{j} \in \mathcal{J}} \alpha_{\boldsymbol{j}} \gamma_{\boldsymbol{j}}(\boldsymbol{u})
$$

integrates to 1 over $\boldsymbol{u} \in \mathbb{R}^{d}$ and is nonnegative. Integration to 1 is justified by the fact that $\gamma_{j_{i}}\left(u_{i}\right)$ is an odd function of $u_{i}$ for each $i=1, \ldots, d$ and the fact that $\int \gamma(\boldsymbol{u}) d \boldsymbol{u}=1$. For nonnegativity of (3.11), note first that the inequality (3.4) implies that, for each $i=1, \ldots, d$,

$$
\left|\gamma_{j_{i}}\left(u_{i}\right)\right|=\sqrt{2}(2 \pi)^{3 / 4} \sqrt{\frac{3^{j_{i}}}{j_{i} !}} \phi\left(u_{i}\right)\left|H_{j_{i}}\left(2 u_{i} / \sqrt{3}\right)\right|
$$




$$
\leq \sqrt{2}(2 \pi)^{1 / 4} 2^{1 / 4} 3^{j_{i} / 2} \exp \left(-\frac{u_{i}^{2}}{6}\right) .
$$

Because we have assumed that $n$ is large enough so that $m \geq 3$, we have $\exp \left(-u_{i}^{2} / 6\right) \leq \exp \left(-u_{i}^{2} /(2 m)\right)$ which gives

$$
\left|\gamma_{j_{i}}\left(u_{i}\right)\right| \leq 8 \cdot 3^{j_{i} / 2} \sqrt{m} \phi_{m}\left(u_{i}\right) \quad \text { for every } i=1, \ldots, d
$$

and consequently

$$
\begin{aligned}
\left|\gamma_{\boldsymbol{j}}(\boldsymbol{u})\right| & \leq 8^{d} 3^{|\boldsymbol{j}| / 2} m^{d / 2} \prod_{i=1}^{d} \phi_{m}\left(u_{i}\right) \\
& =8^{d} 3^{|\boldsymbol{j}| / 2} m^{d / 2} \gamma(\boldsymbol{u}) \text { where }|\boldsymbol{j}|:=j_{1}+\cdots+j_{d} .
\end{aligned}
$$

Thus

$$
\begin{aligned}
\gamma(\boldsymbol{u})+ & \epsilon \sum_{\boldsymbol{j} \in \mathcal{J}} \alpha_{\boldsymbol{j}} \gamma_{\boldsymbol{j}}(\boldsymbol{u}) \geq \gamma(\boldsymbol{u})\left[1-\epsilon 8^{d} m^{d / 2} \sum_{\boldsymbol{j} \in \mathcal{J}} 3^{|\boldsymbol{j}| / 2}\right] \\
& \geq \gamma(\boldsymbol{u})\left[1-\epsilon 8^{d} m^{d / 2} 3^{d(2 m-1) / 2}|\mathcal{J}|\right]=\gamma(\boldsymbol{u})\left[1-\epsilon 8^{d} m^{3 d / 2} 3^{d m}\right]
\end{aligned}
$$

because the maximum value of any $j_{i}$ is $2 m-1 \leq 2 m$ for every $\boldsymbol{j} \in \mathcal{J}$ and the cardinality of $\mathcal{J}$ is $m^{d}$. Plugging in our value of $\epsilon$ (from (3.10)) and using condition (3.9), we get

$$
\gamma(\boldsymbol{u})+\epsilon \sum_{\boldsymbol{j} \in \mathcal{J}} \alpha_{\boldsymbol{j}} \gamma_{\boldsymbol{j}}(\boldsymbol{u}) \geq \gamma(\boldsymbol{u})\left(1-c n^{-1 / 2} m^{5 d / 4} 8^{d} 3^{d m}\right) \geq(1-c) \gamma(\boldsymbol{u})
$$

which implies nonnegativity of (3.11) as long as $c<1$.

We now lower bound $\min _{\boldsymbol{\alpha} \neq \boldsymbol{\beta}} \frac{L^{2}\left(f_{\boldsymbol{\alpha}}, f_{\boldsymbol{\beta}}\right)}{\Upsilon(\boldsymbol{\alpha}, \boldsymbol{\beta})}$ (where $\Upsilon(\boldsymbol{\alpha}, \boldsymbol{\beta}):=\sum_{\boldsymbol{j} \in \mathcal{J}} \mathbb{1}_{\left\{\alpha_{\boldsymbol{j}} \neq \beta_{j}\right\}}$ is the Hamming distance between $\boldsymbol{\alpha}$ and $\boldsymbol{\beta})$. Observe first that

$$
\begin{aligned}
f_{\boldsymbol{\alpha}}(\boldsymbol{x})-f_{\boldsymbol{\beta}}(\boldsymbol{x}) & =\epsilon \int \phi\left(x_{1}-u_{1}\right) \ldots \phi\left(x_{d}-u_{d}\right)\left\{\sum_{\boldsymbol{j} \in \mathcal{J}}\left(\alpha_{\boldsymbol{j}}-\beta_{\boldsymbol{j}}\right) \gamma_{\boldsymbol{j}}(\boldsymbol{u})\right\} d \boldsymbol{u} \\
& =\epsilon \sum_{\boldsymbol{j} \in \mathcal{J}}\left(\alpha_{\boldsymbol{j}}-\beta_{\boldsymbol{j}}\right) \Gamma_{\boldsymbol{j}}(\boldsymbol{x})
\end{aligned}
$$

where

$$
\Gamma_{\boldsymbol{j}}(\boldsymbol{x}):=\int \phi\left(x_{1}-u_{1}\right) \ldots \phi\left(x_{d}-u_{d}\right) \gamma_{\boldsymbol{j}}(\boldsymbol{u}) d \boldsymbol{u} .
$$

As a result

$$
\int\left(f_{\boldsymbol{\alpha}}(\boldsymbol{x})-f_{\boldsymbol{\beta}}(\boldsymbol{x})\right)^{2} d \boldsymbol{x}=\epsilon^{2} \int\left(\sum_{\boldsymbol{j} \in \mathcal{J}}\left(\alpha_{\boldsymbol{j}}-\beta_{\boldsymbol{j}}\right) \Gamma_{\boldsymbol{j}}(\boldsymbol{x})\right)^{2} d \boldsymbol{x}
$$




$$
=\epsilon^{2} \int\left(\sum_{\boldsymbol{j} \in \mathcal{J}}\left(\alpha_{\boldsymbol{j}}-\beta_{\boldsymbol{j}}\right) \mathcal{T}\left(\Gamma_{\boldsymbol{j}}\right)(\boldsymbol{t})\right)^{2} d \boldsymbol{t} .
$$

Because $\Gamma_{j}$ is defined as the product over $i=1, \ldots, d$ of the convolution of $\phi$ and $\gamma_{j_{i}}$, we have

$$
\mathcal{T}\left(\Gamma_{\boldsymbol{j}}\right)(\boldsymbol{t})=\prod_{i=1}^{d} 2^{1 / 2}(2 \pi)^{3 / 4}\left[\sqrt{\frac{3^{j_{i}}}{j_{i} !}} \mathcal{T}(\phi)\left(t_{i}\right) \mathcal{T}\left(\phi(\cdot) H_{j_{i}}\left(\frac{2}{\sqrt{3}} \cdot\right)\right)\left(t_{i}\right)\right] .
$$

By Kim [7, Lemma 2.1], we have

$$
\mathcal{T}\left(\phi(\cdot) H_{k}\left(\frac{2}{\sqrt{3}} \cdot\right)\right)(t)=(-\mathrm{i})^{k} 3^{-k / 2} \phi(t) H_{k}(2 t) \quad \text { for odd } k .
$$

This gives (note that $\sqrt{2}(2 \pi)^{3 / 4} \phi^{2}(t)=\sqrt{2 \phi(2 t)}$ )

$$
\mathcal{T}\left(\Gamma_{\boldsymbol{j}}\right)(\boldsymbol{t})=\prod_{i=1}^{d}(-\mathrm{i})^{j_{i}} \sqrt{2 \phi\left(2 t_{i}\right)} \frac{H_{j_{i}}\left(2 t_{i}\right)}{\sqrt{j_{i} !}}
$$

and thus

$$
\begin{aligned}
& \int\left(f_{\boldsymbol{\alpha}}(\boldsymbol{x})-f_{\boldsymbol{\beta}}(\boldsymbol{x})\right)^{2} d \boldsymbol{x} \\
&=\epsilon^{2} \int\left(\sum_{\boldsymbol{j} \in \mathcal{J}}\left(\alpha_{\boldsymbol{j}}-\beta_{\boldsymbol{j}}\right) \prod_{i=1}^{d}(-\mathrm{i})^{j_{i}} \sqrt{2 \phi\left(2 t_{i}\right)} \frac{H_{j_{i}}\left(2 t_{i}\right)}{\sqrt{j_{i} !}}\right)^{2} d \boldsymbol{t} .
\end{aligned}
$$

The orthogonality of the Hermite polynomials with respect to the weight function $\phi$ (see Equation (3.3)) implies

$$
\int\left(f_{\boldsymbol{\alpha}}(\boldsymbol{x})-f_{\boldsymbol{\beta}}(\boldsymbol{x})\right)^{2} d \boldsymbol{x}=\epsilon^{2} \sum_{\boldsymbol{j} \in \mathcal{J}}\left(\alpha_{\boldsymbol{j}}-\beta_{\boldsymbol{j}}\right)^{2}=\epsilon^{2} \Upsilon(\boldsymbol{\alpha}, \boldsymbol{\beta}) .
$$

We thus have

$$
\min _{\boldsymbol{\alpha} \neq \boldsymbol{\beta}} \frac{L^{2}\left(f_{\boldsymbol{\alpha}}, f_{\boldsymbol{\beta}}\right)}{\Upsilon(\boldsymbol{\alpha}, \boldsymbol{\beta})} \geq \epsilon^{2}
$$

Now we bound the $\chi^{2}$ distance between $f_{\boldsymbol{\alpha}}$ and $f_{\boldsymbol{\beta}}$ for $\boldsymbol{\alpha}$ and $\boldsymbol{\beta}$ with $\Upsilon(\boldsymbol{\alpha}, \boldsymbol{\beta})=1$. Note first that, as a result of (3.13), we have

$$
f_{\boldsymbol{\beta}}(\boldsymbol{x}) \geq(1-c) f_{0}(\boldsymbol{x}) \quad \text { for all } \boldsymbol{\beta}
$$

so that

$$
\chi^{2}\left(f_{\boldsymbol{\alpha}} \| f_{\boldsymbol{\beta}}\right) \leq \frac{1}{1-c} \int \frac{\left(f_{\boldsymbol{\alpha}}-f_{\boldsymbol{\beta}}\right)^{2}}{f_{0}} .
$$


We now split the integral above into $R(\boldsymbol{x}):=\left\{\left|x_{1}\right| \leq M m^{1 / 2}, \ldots,\left|x_{d}\right| \leq\right.$ $\left.M m^{1 / 2}\right\}$ and $R(\boldsymbol{x})^{c}$ where $M$ is a dimensional constant larger than $8 d \log 3$. Then using $f_{0}(\boldsymbol{x})\{x \in R(\boldsymbol{x})\} \geq m^{-d / 2} / C_{d}$ (for some dimensional constant $C_{d}$ ), we have

$$
\begin{aligned}
& (1-c) \chi^{2}\left(f_{\boldsymbol{\alpha}} \| f_{\boldsymbol{\beta}}\right) \leq \int \frac{\left(f_{\boldsymbol{\alpha}}(\boldsymbol{x})-f_{\boldsymbol{\beta}}(\boldsymbol{x})\right)^{2}}{f_{0}(\boldsymbol{x})} \\
& \leq C_{d} m^{d / 2} \int\left(f_{\boldsymbol{\alpha}}(\boldsymbol{x})-f_{\boldsymbol{\beta}}(\boldsymbol{x})\right)^{2}+\int_{R(x)^{c}} \frac{\left(f_{\boldsymbol{\alpha}}(\boldsymbol{x})-f_{\boldsymbol{\beta}}(\boldsymbol{x})\right)^{2}}{f_{0}(\boldsymbol{x})} d \boldsymbol{x} \\
& =C_{d} m^{d / 2} \epsilon^{2}+\int_{R(x)^{c}} \frac{\left(f_{\boldsymbol{\alpha}}(\boldsymbol{x})-f_{\boldsymbol{\beta}}(\boldsymbol{x})\right)^{2}}{f_{0}(\boldsymbol{x})} d \boldsymbol{x} \leq \frac{1}{2 n}+\int_{R(x)^{c}} \frac{\left(f_{\boldsymbol{\alpha}}(\boldsymbol{x})-f_{\boldsymbol{\beta}}(\boldsymbol{x})\right)^{2}}{f_{0}(\boldsymbol{x})} d \boldsymbol{x}
\end{aligned}
$$

provided $c$ is chosen above so that $c^{2} C_{d} \leq 1 / 2$ (recall that $\epsilon^{2}=c^{2} n^{-1} m^{-d / 2}$ ). The second term above is bounded as follows. Denoting $\boldsymbol{j}^{*}$ by the index where $\boldsymbol{\alpha}$ and $\boldsymbol{\beta}$ differ so that $\alpha_{\boldsymbol{j}^{*}} \neq \beta_{\boldsymbol{j}^{*}}$ (recall that $\Upsilon(\boldsymbol{\alpha}, \boldsymbol{\beta})=1$ ), we get by (3.12),

$$
\begin{aligned}
\int_{R(x)^{c}} & \frac{\left(f_{\boldsymbol{\alpha}}(\boldsymbol{x})-f_{\boldsymbol{\beta}}(\boldsymbol{x})\right)^{2}}{f_{0}(\boldsymbol{x})} d \boldsymbol{x} \\
& \leq \epsilon^{2} 3^{d / 2}(4 \pi)^{3 / 4} 3^{\left|\boldsymbol{j}^{*}\right|} \int_{R(x)^{c}} \frac{\left(\int \phi\left(x_{1}-u_{1}\right) \ldots \phi\left(x_{d}-u_{d}\right) \boldsymbol{\phi}_{3}(\boldsymbol{u}) d \boldsymbol{u}\right)^{2}}{f_{0}(\boldsymbol{x})} d \boldsymbol{x} \\
& \leq d \epsilon^{2} 3^{d / 2}(4 \pi)^{3 / 4} 3^{\left|\boldsymbol{j}^{*}\right|} \int_{\left\{\left|x_{1}\right|>M \sqrt{m}\right\}} \frac{\boldsymbol{\phi}_{4}^{2}(\boldsymbol{x})}{\boldsymbol{\phi}_{1+m}(\boldsymbol{x})} d \boldsymbol{x} \\
& \leq 2 d c^{2} n^{-1} 3^{d / 2}(4 \pi)^{3 / 4} 3^{\left|\boldsymbol{j}^{*}\right|} \int_{\left\{x_{1}>M \sqrt{m}\right\}} \phi_{4}\left(x_{1}\right) d x_{1} \leq \frac{1}{2 n}
\end{aligned}
$$

where the second inequality follows by bounding the maximum by the sum with symmetry, the penultimate inequality follows first by plugging (3.10) in $\epsilon^{2}$ and then since $\phi_{1+m}(\boldsymbol{x}) \geq m^{-d / 2} \boldsymbol{\phi}_{4}(\boldsymbol{x})$ and $3^{\left|\boldsymbol{j}^{*}\right|} \int_{\left\{x_{1}>M m^{1 / 2}\right\}} \phi_{4}\left(x_{1}\right) d x_{1} \leq 1$ by taking $M$ larger than $8 d \log 3$, and the last inequality follows by choosing $c$ to be a small enough dimensional constant such that $c^{2} \leq(4 \pi)^{-3 / 4} 3^{-d / 2} /(2 d)$.

By Assouad's lemma 3.1, we have

$$
R_{n}\left(\mathcal{F}_{d}, L^{2}\right) \geq \frac{m^{d}}{8} \epsilon^{2}\left(1-\sqrt{\frac{1}{2(1-c)}}\right)
$$

Of course $c$ can be taken to be small enough so that the right hand side above is larger than a constant (depending on $d$ alone) multiple of $(\log n)^{d / 2} / n$. The proof is thus complete.

\subsection{Proof of Theorem 2.2}

The idea for the lower bound on $R_{n}\left(\mathcal{F}_{\mathcal{G}_{1}(\Gamma)}, h^{2}\right)$ is to again construct a subset of $\mathcal{F}_{\mathcal{G}_{1}(\Gamma)}$ indexed by a hypercube and then use Assouad's lemma. Our construction 
is a natural extension to $d \geq 1$ of the one-dimensional construction in [7]. Let $\sigma^{2}=\min (\Gamma, 1)$. Let $m$ be the largest integer such that

$$
\frac{m^{d} 4^{d}\left(\frac{9}{\sigma^{2}}\right)^{d m}}{\sqrt{n}} \leq 1
$$

The above condition implies $m \leq \frac{\log n}{2 d \log \left(9 / \sigma^{2}\right)}$ and $m \geq \frac{\log n}{4 d \log \left(9 / \sigma^{2}\right)}$. In order to use Assouad's lemma, we construct densities via perturbations of the density

$$
f_{0}(\boldsymbol{x})=\phi_{1+\sigma^{2}}\left(x_{1}\right) \ldots \phi_{1+\sigma^{2}}\left(x_{d}\right)
$$

where, it may be recalled, $\phi_{\sigma^{2}}(\cdot)$ denotes the univariate normal density with mean zero and variance $\sigma^{2}$. Note that $f_{0}$ can also be written as

$f_{0}(\boldsymbol{x})=\int \phi\left(x_{1}-u_{1}\right) \ldots \phi\left(x_{d}-u_{d}\right) v(\boldsymbol{u}) d \boldsymbol{u} \quad$ where $v(\boldsymbol{u}):=\phi_{\sigma^{2}}\left(u_{1}\right) \ldots \phi_{\sigma^{2}}\left(u_{d}\right)$.

Again we let

$$
\mathcal{J}:=\{1,3, \ldots, 2 m-1\}^{d}
$$

with $N:=m^{d}$ and we consider index binary vectors in $\{0,1\}^{N}$ by elements $\boldsymbol{j}:=\left(j_{1}, \ldots, j_{d}\right)$ of $\mathcal{J}$. For $\boldsymbol{\alpha}=\left(\alpha_{\boldsymbol{j}}, \boldsymbol{j} \in \mathcal{J}\right)$, we let

$$
f_{\boldsymbol{\alpha}}(\boldsymbol{x})=\int \phi\left(x_{1}-u_{1}\right) \ldots \phi\left(x_{d}-u_{d}\right)\left[v(\boldsymbol{u})+\epsilon \sum_{\boldsymbol{j} \in \mathcal{J}} \alpha_{\boldsymbol{j}} v_{\boldsymbol{j}}(\boldsymbol{u})\right] d \boldsymbol{u}
$$

where $\epsilon$ is given by

$$
\epsilon=\frac{1}{2} n^{-1 / 2}
$$

and

$$
v_{\boldsymbol{j}}(\boldsymbol{u}):=v_{j_{1}}\left(u_{1}\right) \ldots v_{j_{d}}\left(u_{d}\right) \quad \text { with } v_{j_{i}}\left(u_{i}\right)=\frac{C_{j_{i}}}{\sqrt{j_{i} !}} \phi\left(\rho u_{i}\right) H_{j_{i}}\left(\gamma u_{i}\right)
$$

where we define

$$
\begin{aligned}
\varphi & =1+\frac{1}{1+2 \sigma^{2}} \\
\rho & =\left(\varphi-1+\frac{\left(1+\sigma^{2} \varphi\right)^{1 / 2}+1}{\sigma^{2}}\right)^{1 / 2} \\
\gamma & =\sqrt{2} \frac{\left(1+\sigma^{2} \varphi\right)^{1 / 4}}{\sigma} \\
C_{j_{i}} & =(2 \pi)^{-1 / 4} \varphi^{1 / 2}\left(1+\sigma^{2} \varphi\right)^{1 / 4} \frac{\left(\frac{\left(1+\sigma^{2} \varphi\right)^{1 / 2}+1}{\left(1+\sigma^{2} \varphi\right)^{1 / 2}-1}\right)^{j_{i} / 2}}{\left(\left(1+\sigma^{2} \varphi\right)^{1 / 2}-1\right)^{1 / 2}} .
\end{aligned}
$$


By an analogous argument in the proof of Theorem 2.1, we can show $f_{\boldsymbol{\alpha}} \in$ $\mathcal{F}_{\mathcal{G}_{1}(\Gamma)}$. First, integration of $f_{\boldsymbol{\alpha}}(\boldsymbol{x})$ to 1 is guaranteed since $v_{j_{i}}\left(u_{i}\right)$ is an odd function of $u_{i}$ for each $i=1, \ldots, d$ and the fact that $\int v(\boldsymbol{u}) d \boldsymbol{u}=1$. For nonnegativity of $v(\boldsymbol{u})+\epsilon \sum_{\boldsymbol{j} \in \mathcal{J}} \alpha_{\boldsymbol{j}} v_{\boldsymbol{j}}(\boldsymbol{u})$, note that using (3.4) we have

$$
\left|v_{j_{i}}\left(u_{i}\right)\right| \leq \frac{\kappa C_{j_{i}}}{\sqrt{2 \pi}} \exp \left(-\frac{1}{2} u_{i}^{2}\left(\rho^{2}-\frac{1}{2} \gamma^{2}\right)\right)
$$

where

$$
\rho^{2}-\frac{1}{2} \gamma^{2}=\frac{1}{1+2 \sigma^{2}}+\frac{1}{\sigma^{2}} \geq \frac{1}{\sigma^{2}}
$$

Hence

$$
\left|v_{j_{i}}\left(u_{i}\right)\right| \leq \kappa C_{j_{i}} \sigma \phi_{\sigma^{2}}\left(u_{i}\right)
$$

for every $i=1, \ldots, d$. Thus

$$
\left|v_{\boldsymbol{j}}(\boldsymbol{u})\right| \leq(\sigma \kappa)^{d}\left(\prod_{i=1}^{d} C_{j_{i}}\right) v(\boldsymbol{u}) \leq 4^{d}\left(\frac{3}{\sigma}\right)^{|\boldsymbol{j}|} v(\boldsymbol{u})
$$

where the last inequality follows by

$$
\left(\frac{\left(1+\sigma^{2} \varphi\right)^{1 / 2}+1}{\left(1+\sigma^{2} \varphi\right)^{1 / 2}-1}\right)^{1 / 2}=\frac{\left(1+\sigma^{2} \varphi\right)^{1 / 2}+1}{\sigma \varphi^{1 / 2}} \leq \frac{3}{\sigma}
$$

and

$$
\sigma \leq 2\left(\left(1+\sigma^{2} \varphi\right)^{1 / 2}-1\right)^{1 / 2}
$$

which gives

$$
\begin{aligned}
v(\boldsymbol{u})+\epsilon \sum_{\boldsymbol{j} \in \mathcal{J}} \alpha_{\boldsymbol{j}} v_{\boldsymbol{j}}(\boldsymbol{u}) & \geq v(\boldsymbol{u})\left[1-\epsilon 4^{d} \sum_{\boldsymbol{j} \in \mathcal{J}}\left(\frac{3}{\sigma}\right)^{|\boldsymbol{j}|}\right] \\
& \geq v(\boldsymbol{u})\left[1-\epsilon 4^{d} m^{d}\left(\frac{9}{\sigma^{2}}\right)^{d m}\right]
\end{aligned}
$$

Plugging in $\epsilon=n^{-1 / 2} / 2$ from (3.14),

$$
v(\boldsymbol{u})+\epsilon \sum_{\boldsymbol{j} \in \mathcal{J}} \alpha_{\boldsymbol{j}} v_{\boldsymbol{j}}(\boldsymbol{u}) \geq v(\boldsymbol{u})\left(1-\frac{1}{2} n^{-1 / 2} 4^{d} m^{d}\left(\frac{9}{\sigma^{2}}\right)^{d m}\right) \geq \frac{1}{2} v(\boldsymbol{u})>0
$$

In the same way, we have

$$
v(\boldsymbol{u})+\epsilon \sum_{\boldsymbol{j} \in \mathcal{J}} \alpha_{\boldsymbol{j}} v_{\boldsymbol{j}}(\boldsymbol{u}) \leq \frac{3}{2} v(\boldsymbol{u})
$$


To claim $f_{\alpha} \in \mathcal{F}_{\mathcal{G}_{1}(\Gamma)}$, we need to show $v(\boldsymbol{u})+\epsilon \sum_{\boldsymbol{j} \in \mathcal{J}} \alpha_{\boldsymbol{j}} v_{\boldsymbol{j}}(\boldsymbol{u})$ is sub-gaussian. Indeed,

$$
\begin{aligned}
\int_{\|u\|>t} v(\boldsymbol{u})+\epsilon \sum_{j \in \mathcal{J}} \alpha_{\boldsymbol{j}} v_{\boldsymbol{j}}(\boldsymbol{u}) d \boldsymbol{u} & \leq \frac{3}{2} \int_{\|u\|>t} \phi_{\sigma^{2}}\left(u_{1}\right) \ldots \phi_{\sigma^{2}}\left(u_{d}\right) d \boldsymbol{u} \\
& \leq C \exp \left(-t^{2} / 2 \sigma^{2}\right) \leq C \exp \left(-t^{2} / 2 \Gamma\right)
\end{aligned}
$$

where $C$ is a constant depending on $d$ and the last inequality follows by the choice of $\sigma^{2}=\min (\Gamma, 1)$.

The fact that all these constructed mixing densities are between $(1 / 2) v(\boldsymbol{u})$ and $(3 / 2) v(\boldsymbol{u})$ gives

$$
\frac{1}{2} f_{0}(\boldsymbol{x}) \leq f_{\boldsymbol{\alpha}}(\boldsymbol{x}) \leq \frac{3}{2} f_{0}(\boldsymbol{x}) .
$$

Inequality (3.15) implies that

$$
h^{2}\left(f_{\boldsymbol{\alpha}}, f_{\boldsymbol{\beta}}\right) \geq \frac{1}{6} \int \frac{\left(f_{\boldsymbol{\alpha}}-f_{\boldsymbol{\beta}}\right)^{2}}{f_{0}} \quad \text { and } \quad \chi^{2}\left(f_{\boldsymbol{\alpha}} \| f_{\boldsymbol{\beta}}\right) \leq 2 \int \frac{\left(f_{\boldsymbol{\alpha}}-f_{\boldsymbol{\beta}}\right)^{2}}{f_{0}}
$$

because, respectively,

$$
h^{2}\left(f_{\boldsymbol{\alpha}}, f_{\boldsymbol{\beta}}\right)=\int \frac{\left(f_{\boldsymbol{\alpha}}-f_{\boldsymbol{\beta}}\right)^{2}}{\left(\sqrt{f_{\boldsymbol{\alpha}}}+\sqrt{f_{\boldsymbol{\beta}}}\right)^{2}} \geq \frac{1}{6} \int \frac{\left(f_{\boldsymbol{\alpha}}-f_{\boldsymbol{\beta}}\right)^{2}}{f_{0}}
$$

and

$$
\chi^{2}\left(f_{\boldsymbol{\alpha}} \| f_{\boldsymbol{\beta}}\right)=\int \frac{\left(f_{\boldsymbol{\alpha}}-f_{\boldsymbol{\beta}}\right)^{2}}{f_{\boldsymbol{\alpha}}} \leq 2 \int \frac{\left(f_{\boldsymbol{\alpha}}-f_{\boldsymbol{\beta}}\right)^{2}}{f_{0}} .
$$

By inequality (3.16), it is clear that for the application of Assouad's Lemma 3.1, it is enough to focus on the quantity $\int \frac{\left(f_{\boldsymbol{\alpha}}-f_{\boldsymbol{\beta}}\right)^{2}}{f_{0}}$. Let us write

$$
\Lambda_{\boldsymbol{j}}:=\int \phi\left(x_{1}-u_{1}\right) \ldots \phi\left(x_{d}-u_{d}\right) v_{\boldsymbol{j}}(\boldsymbol{u}) d \boldsymbol{u}
$$

and we consider

$$
\begin{aligned}
\int \frac{\left(f_{\boldsymbol{\alpha}}-f_{\boldsymbol{\beta}}\right)^{2}}{f_{0}} & =\epsilon^{2} \int\left(\sum_{\boldsymbol{j} \in \mathcal{J}}\left(\alpha_{\boldsymbol{j}}-\beta_{\boldsymbol{j}}\right) \frac{\Lambda_{\boldsymbol{j}}}{\sqrt{f_{0}}}\right)^{2} \\
& =\epsilon^{2} \int\left(\sum_{\boldsymbol{j} \in \mathcal{J}}\left(\alpha_{\boldsymbol{j}}-\beta_{\boldsymbol{j}}\right) \mathcal{T}\left(\frac{\Lambda_{\boldsymbol{j}}}{\sqrt{f_{0}}}\right)\right)^{2} .
\end{aligned}
$$

Because $\Lambda_{j} / \sqrt{f_{0}}$ is defined as the product over $i=1, \ldots, d$, of

$$
\frac{\int \phi\left(x_{i}-u_{i}\right) v_{j_{i}}\left(u_{i}\right) d u_{i}}{\sqrt{\int \phi\left(x_{i}-u_{i}\right) \phi_{\sigma^{2}}\left(u_{i}\right) d u_{i}}}
$$




$$
=\frac{C_{j_{i}}(4 \pi)^{1 / 4}}{\sqrt{j_{i} ! \varphi}} \int \phi_{\varphi}\left(x_{i}-u_{i}\right) \phi\left(\frac{\sqrt{\rho^{2}+1-\varphi}}{\varphi} u_{i}\right) H_{j_{i}}\left(\frac{\gamma}{\varphi} u_{i}\right),
$$

where the above equality follows by Kim [7, Lemma 2.2], we have

$$
\mathcal{T}\left(\frac{\Lambda_{j}}{\sqrt{f_{0}}}\right)\left(t_{i}\right)=\prod_{i=1}^{d} \frac{C_{j_{i}}(4 \pi)^{1 / 4}}{\sqrt{j_{i} ! \varphi}} \mathcal{T}\left(\phi_{\varphi}\right)\left(t_{i}\right) \mathcal{T}\left(\phi\left(\frac{\sqrt{\rho^{2}+1-\varphi}}{\varphi} \cdot\right) H_{j_{i}}\left(\frac{\gamma}{\varphi} \cdot\right)\right)\left(t_{i}\right) .
$$

By Kim [7, Lemma 2.1], we have by denoting $\varrho=\sqrt{2}\left(1+\sigma^{2} \varphi\right)^{1 / 4} \varphi^{1 / 2}$,

$$
\begin{aligned}
& \mathcal{T}\left(\phi\left(\frac{\sqrt{\rho^{2}+1-\varphi}}{\varphi} \cdot\right) H_{j_{i}}\left(\frac{\gamma}{\varphi} \cdot\right)\right)\left(t_{i}\right) \\
& \quad=(-\mathrm{i})^{j_{i}} \frac{\sigma \varphi}{\left(\left(1+\sigma^{2} \varphi\right)^{1 / 2}+1\right)^{1 / 2}}\left(\frac{\left(1+\sigma^{2} \varphi\right)^{1 / 2}-1}{\left(1+\sigma^{2} \varphi\right)^{1 / 2}+1}\right)^{j_{i} / 2} \times \\
& \phi\left(\frac{\sigma \varphi}{\left(\left(1+\sigma^{2} \varphi\right)^{1 / 2}+1\right)^{1 / 2}} t_{i}\right) H_{j_{i}}\left(\varrho t_{i}\right) .
\end{aligned}
$$

Using

$$
\mathcal{T}\left(\phi_{\varphi}\right)\left(t_{i}\right)=\frac{1}{\sqrt{2 \pi}} \exp \left(-\frac{\varphi}{2} t_{i}^{2}\right)
$$

we have

$$
\mathcal{T}\left(\frac{\Lambda_{j}}{\sqrt{f_{0}}}\right)=\prod_{i=1}^{d}(-\mathrm{i})^{j_{i}} \sqrt{\varrho \phi\left(\varrho t_{i}\right)} \frac{H_{j_{i}}\left(\varrho t_{i}\right)}{\sqrt{j_{i} !}} .
$$

Consequently,

$$
\int \frac{\left(f_{\boldsymbol{\alpha}}-f_{\boldsymbol{\beta}}\right)^{2}}{f_{0}}=\epsilon^{2} \Upsilon(\boldsymbol{\alpha}, \boldsymbol{\beta}) .
$$

This (and (3.16)) gives

$$
\min _{\boldsymbol{\alpha} \neq \boldsymbol{\beta}} \frac{h^{2}\left(f_{\boldsymbol{\alpha}}, f_{\boldsymbol{\beta}}\right)}{\Upsilon(\boldsymbol{\alpha}, \boldsymbol{\beta})} \geq \frac{1}{6} \epsilon^{2} \quad \text { and } \quad \chi^{2}\left(f_{\boldsymbol{\alpha}}, f_{\boldsymbol{\beta}}\right) \leq 2 \epsilon^{2} .
$$

By Lemma 3.1, we have

$$
R_{n}\left(\mathcal{F}_{\mathcal{G}_{1}(\Gamma)}, h^{2}\right) \geq \frac{m^{d}}{8} \frac{(1 / 2)^{2}}{6 n}\left(\frac{1}{2}\right) \geq c_{d, \Gamma} \frac{(\log n)^{d}}{n},
$$

where $c_{d, \Gamma}=\frac{1}{384}\left(\frac{1}{4 d \log \left(9 / \min \left(1, \Gamma^{2}\right)\right)}\right)^{d}$.

\subsection{Proof of Theorem 2.3}

The idea for the lower bound on $R_{n}\left(\mathcal{F}_{\mathcal{G}_{2}(p, K)}, h^{2}\right)$ is again to construct a subset of $\mathcal{F}_{\mathcal{G}_{2}(p, K)}$ indexed by a hypercube and then we use Assouad's lemma. Let $a_{0}=\min \left\{(K / d) 7^{-1 / p}, 1\right\}$ and

$$
S:=\left\{a_{0}+(\ell-1) M \log (\ell e), 1 \leq \ell \leq k_{0}\right\}
$$


where

$$
M=C_{d, p} \sqrt{\log n} \quad \text { with } C_{d, p}=\sqrt{8\left(\frac{2 d}{p+d}+1\right)}
$$

and $k_{0}$ the largest integer less than or equal to $k_{0}^{\prime}$ where

$$
k_{0}^{\prime}=c n^{1 /(p+d)}(\log n)^{-3 / 2} \quad \text { with } c=40^{-1 / 2} a_{0}^{1+2 /(p+d)} .
$$

Let $k=k_{0}^{d}$ and let $\mathbf{a}_{1}, \ldots, \mathbf{a}_{k}$ be an enumeration of the points in $S^{d}=S \times$ $\cdots \times S$. We can take $\mathbf{a}_{1}=\left(a_{0}, a_{0}, \ldots, a_{0}\right) \in \mathbb{R}^{d}, \mathbf{a}_{2}=\left(a_{0}+M \log (2 e), 1, \ldots, 1\right) \in$ $\mathbb{R}^{d}, \ldots, \mathbf{a}_{k}=\left(a_{0}+\left(k_{0}-1\right) M \log \left(k_{0} e\right), \ldots, a_{0}+\left(k_{0}-1\right) M \log \left(k_{0} e\right)\right) \in \mathbb{R}^{d}$. Let $G$ be the discrete probability distribution supported on $S^{d}$ that is given by

$$
G\left\{\mathbf{a}_{i}\right\}=C_{n, d, p}\left(\left\langle\mathbf{a}_{i}, \mathbf{1}\right\rangle-d a_{0}+a_{0}\right)^{-(p+d)} \quad \text { for } i=1, \ldots, k
$$

where $\mathbf{1}$ is the $d$-dimensional vector of ones and

$$
C_{n, d, p}:=\frac{1}{\sum_{\ell_{1}=1}^{k_{0}} \cdots \sum_{\ell_{d}=1}^{k_{0}}\left(a_{0}+\sum_{j=1}^{d}\left(\left(\ell_{j}-1\right) M \log \left(\ell_{j} e\right)\right)\right)^{-(p+d)}}
$$

is the normalizing constant. We assume $n$ is sufficiently large so that $\log \log k_{0} \leq$ $M$ and $M p \geq 2^{d}$. We claim that

$$
a_{0}^{p+d} / 2<C_{n, d, p}<a_{0}^{p+d} \quad \text { and } \quad G \in \mathcal{G}_{2}\left(p, 6^{1 / p} d a_{0}\right) .
$$

Let us assume the above claim for now and proceed with the proof. The claim will be proved later. For $i=1, \ldots, k$, let $\mathbf{b}_{i}=\mathbf{a}_{i}+\left(\delta_{i} / \sqrt{d}\right) \mathbf{1}$ where

$$
\delta_{i}=\frac{1}{\sqrt{2 n G\left(\mathbf{a}_{i}\right)}}
$$

The choice of $k_{0}$ means that $\left\langle\mathbf{a}_{i}, \mathbf{1}\right\rangle \leq d a_{0}-d M \log \left(k_{0} e\right)+d k_{0} M \log \left(k_{0} e\right) \leq$ $d a_{0}-a_{0}+n^{1 /(p+d)} a_{0}^{1+2 /(p+d)}$, which implies that $G\left(\mathbf{a}_{i}\right)>a_{0}^{-2}(2 n)^{-1}$ for every $i=1, \ldots, k$. Hence $0<\delta_{i}<a_{0}$ (thus $0<\delta_{i}<1$ ).

Note that $\left\|\mathbf{b}_{i}-\mathbf{a}_{i}\right\|=\delta_{i}$ for all $i$. Define, for every $\boldsymbol{\alpha}=\left(\alpha_{1}, \ldots, \alpha_{k}\right) \in\{0,1\}^{k}$,

$$
f_{\boldsymbol{\alpha}}(\boldsymbol{x})=\sum_{i=1}^{k} G\left(\mathbf{a}_{i}\right) \phi_{d}\left(\boldsymbol{x}-\mathbf{a}_{i}\left(1-\alpha_{i}\right)-\mathbf{b}_{i} \alpha_{i}\right)
$$

where $\phi_{d}$ is the standard normal density on $\mathbb{R}^{d}$. Since $G \in \mathcal{G}_{2}\left(p, 6^{1 / p} d a_{0}\right)$ and $\left\|\mathbf{b}_{i}-\mathbf{a}_{i}\right\|^{p}=\delta_{i}^{p} d^{1-p / 2}$ for every $i=1, \ldots, k$,

$\sum_{i=1}^{k}\left\|\mathbf{a}_{i}+\alpha_{i}\left(\mathbf{b}_{i}-\mathbf{a}_{i}\right)\right\|^{p} G\left\{\mathbf{a}_{i}\right\} \leq 2^{p-1} \int\left(\|u\|^{p}+d^{1-p / 2} a_{0}^{p}\right) d G \leq 7\left(d a_{0}\right)^{p} \leq K^{p}$

by definition of $a_{0}$. This shows $f_{\boldsymbol{\alpha}} \in \mathcal{F}_{\mathcal{G}_{2}(p, K)}$. 
We shall use Assouad's lemma for the class $f_{\boldsymbol{\alpha}}, \boldsymbol{\alpha} \in\{0,1\}^{k}$. For fixed $\boldsymbol{\alpha}=$ $\left(\alpha_{1}, \ldots, \alpha_{k}\right)$ and $\boldsymbol{\beta}=\left(\beta_{1}, \ldots, \beta_{k}\right)$ in $\{0,1\}^{k}$, we can write (with $f_{i}(x):=\phi_{d}(x-$ $\left.a_{i}\left(1-\alpha_{i}\right)-b_{i} \alpha_{i}\right)$ and $\left.g_{j}(x):=\phi_{d}\left(x-a_{j}\left(1-\beta_{j}\right)-b_{j} \beta_{j}\right)\right)$

$$
\begin{aligned}
h^{2}\left(f_{\boldsymbol{\alpha}}, f_{\boldsymbol{\beta}}\right) & =2\left(1-\int \sqrt{f_{\boldsymbol{\alpha}}(\boldsymbol{x}) f_{\boldsymbol{\beta}}(\boldsymbol{x})} d \boldsymbol{x}\right) \\
& =2\left(1-\int \sqrt{\sum_{i, j} G\left(\mathbf{a}_{i}\right) G\left(\mathbf{a}_{j}\right) f_{i}(\boldsymbol{x}) g_{j}(\boldsymbol{x})} d \boldsymbol{x}\right) \\
& \geq 2\left(1-\sum_{i, j} \sqrt{G\left(\mathbf{a}_{i}\right) G\left(\mathbf{a}_{j}\right)} \int \sqrt{f_{i}(\boldsymbol{x}) g_{j}(\boldsymbol{x})} d \boldsymbol{x}\right)
\end{aligned}
$$

where the last inequality follows from $\sqrt{\sum_{i} x_{i}} \leq \sum_{i} \sqrt{x_{i}}$ for $x_{i} \geq 0$. Saha and Guntuboyina [11, Equation (A.18)] now gives

$$
\begin{aligned}
& \frac{1}{2} h^{2}\left(f_{\boldsymbol{\alpha}}, f_{\boldsymbol{\beta}}\right) \\
& =\left(1-\sum_{i} G\left(\mathbf{a}_{i}\right) \int \sqrt{f_{i}(\boldsymbol{x}) g_{i}(\boldsymbol{x})} d \boldsymbol{x}-\sum_{i \neq j} \sqrt{G\left(\mathbf{a}_{i}\right) G\left(\mathbf{a}_{j}\right)} \int \sqrt{f_{i}(\boldsymbol{x}) g_{j}(\boldsymbol{x})} d \boldsymbol{x}\right) \\
& \geq \sum_{i=1}^{k} G\left(\mathbf{a}_{i}\right)\left|\alpha_{i}-\beta_{i}\right|\left(1-e^{-\delta_{i}^{2} / 8}\right)-\sum_{i \neq j} \sqrt{G\left(\mathbf{a}_{i}\right) G\left(\mathbf{a}_{j}\right)} e^{-M^{2} / 8} \\
& \geq \sum_{i=1}^{k} G\left(\mathbf{a}_{i}\right) \frac{\delta_{i}^{2}}{10}\left|\alpha_{i}-\beta_{i}\right|-\frac{1}{40 n},
\end{aligned}
$$

where the last inequality follows since $1-e^{-a^{2} / 8} \geq a^{2} / 10$ for every $0<a<1$, and

$$
\begin{aligned}
\sum_{i \neq j} \sqrt{G\left(\mathbf{a}_{i}\right) G\left(\mathbf{a}_{j}\right)} e^{-M^{2} / 8} & \leq k^{2} e^{-M^{2} / 8}=k_{0}^{2 d} e^{-C_{d, p}^{2} \log n / 8} \\
& \leq(1 / \sqrt{40})^{2 d} n^{\frac{2 d}{p+d}-\frac{C_{d, p}^{2}}{8}} \leq \frac{1}{40 n}
\end{aligned}
$$

by the choice of $k_{0}$ and $C_{d, p}$. This implies, by the choice of $\delta_{i}^{2}=1 /\left(2 n G\left(\mathbf{a}_{i}\right)\right)$, that

$$
h^{2}\left(f_{\boldsymbol{\alpha}}, f_{\boldsymbol{\beta}}\right) \geq \frac{\sum_{i=1}^{k}\left|\alpha_{i}-\beta_{i}\right|}{20 n}-\frac{1}{40 n} \geq \frac{\Upsilon(\boldsymbol{\alpha}, \boldsymbol{\beta})}{40 n} .
$$

Now suppose that $\Upsilon(\boldsymbol{\alpha}, \boldsymbol{\beta})=1$ and let $l$ be the unique index such that $\alpha_{l} \neq \beta_{l}$. Then for $\delta_{l}^{2} \leq 1$,

$$
\chi^{2}\left(f_{\boldsymbol{\alpha}} \| f_{\boldsymbol{\beta}}\right)=\int \frac{\left(f_{\boldsymbol{\alpha}}(\boldsymbol{x})-f_{\boldsymbol{\beta}}(\boldsymbol{x})\right)^{2}}{f_{\boldsymbol{\alpha}}(\boldsymbol{x})} d \boldsymbol{x}
$$




$$
\begin{aligned}
& =\int \frac{\left(\sum_{i=1}^{k} G\left(\mathbf{a}_{i}\right) f_{i}(\boldsymbol{x})-\sum_{i=1}^{k} G\left(\mathbf{a}_{i}\right) g_{i}(\boldsymbol{x})\right)^{2}}{\sum_{i=1}^{k} G\left(\mathbf{a}_{i}\right) f_{i}(\boldsymbol{x})} d \boldsymbol{x} \\
& \leq \int \frac{G\left(\mathbf{a}_{l}\right)^{2}\left(f_{l}(\boldsymbol{x})-g_{l}(\boldsymbol{x})\right)^{2}}{G\left(\mathbf{a}_{l}\right) f_{l}(\boldsymbol{x})} d \boldsymbol{x}=G\left(\mathbf{a}_{l}\right)\left(\int \frac{g_{l}^{2}(\boldsymbol{x})}{f_{l}(\boldsymbol{x})} d \boldsymbol{x}-1\right) \\
& \leq G\left(\mathbf{a}_{l}\right)\left(e^{\delta_{l}^{2}}-1\right) \leq 2 G\left(\mathbf{a}_{l}\right) \delta_{l}^{2}=\frac{1}{n},
\end{aligned}
$$

where the penultimate inequality follows since

$$
\int \frac{g_{l}^{2}(\boldsymbol{x})}{f_{l}(\boldsymbol{x})} d \boldsymbol{x}=\int \frac{\phi_{d}^{2}\left(\boldsymbol{x}-\mathbf{a}_{l}\left(1-\beta_{l}\right)-\mathbf{b}_{l} \beta_{l}\right)}{\phi_{d}\left(\boldsymbol{x}-\mathbf{a}_{l}\left(1-\alpha_{l}\right)-\mathbf{b}_{l} \alpha_{l}\right)} d \boldsymbol{x} \leq \exp \left(\left\|\mathbf{a}_{l}-\mathbf{b}_{l}\right\|^{2}\right)=\exp \left(\delta_{l}^{2}\right),
$$

and the last inequality follows since $e^{a} \leq 2 a+1$ when $0 \leq a \leq 1$.

By Lemma 3.1, we have

$$
R_{n}\left(\mathcal{F}_{\mathcal{G}_{2}(p, K)}, h^{2}\right) \geq \frac{k}{8} \frac{1}{40 n}\left(1-\sqrt{\frac{1}{2}}\right) \geq c_{d, p, K} n^{-p /(p+d)}(\log n)^{-3 d / 2}
$$

where $c_{d, p, K}=\frac{1}{320}(1-\sqrt{1 / 2}) 40^{-d / 2}\left\{\min \left((K / d) 7^{-1 / p}, 1\right)\right\}^{d+2 d /(p+d)}$, a constant only depending on $d, p$ and $K$ (but not a function of $n$ ).

It remains to prove the claim (3.20). Let us first show that $a_{0}^{p+d} / 2<C_{n, d, p}<$ $a_{0}^{p+d}$. The definition (3.19) immediately gives $C_{n, d, p}<a_{0}^{p+d}$ so we only need to show that $C_{n, d, p}>a_{0}^{p+d} / 2$. For this, let us bound the denominator of $C_{n, d, p}$ using the inequality

$$
\begin{aligned}
\sum_{\ell_{1}=1}^{k_{0}} \cdots \sum_{\ell_{d}=1}^{k_{0}} & \left(a_{0}+\sum_{j=1}^{d}\left(\ell_{j}-1\right) M\right)^{-(p+d)} \\
& \leq \sum_{\ell_{1}=1}^{k_{0}}\left(a_{0}+\left(\ell_{1}-1\right) M\right)^{-(p+1)} \prod_{j=2}^{d}\left(a_{0}^{-1}+\frac{1}{(p+j-1) M}\right)
\end{aligned}
$$

The above inequality will be proved at the end of the proof. The above inequality, along with the inequality

$$
\begin{aligned}
\sum_{\ell=1}^{k_{0}}\left(a_{0}+\right. & (\ell-1) M)^{-(p+1)} \leq a_{0}^{-(p+1)}+\int_{1}^{k_{0}}\left(a_{0}+(x-1) M\right)^{-(p+1)} d x \\
& =a_{0}^{-p}\left(a_{0}^{-1}+\frac{1}{p M}\left(1-\left(\frac{a_{0}}{a_{0}+\left(k_{0}-1\right) M}\right)^{p}\right)\right) \leq a_{0}^{-p}\left(a_{0}^{-1}+\frac{1}{p M}\right)
\end{aligned}
$$

imply that the denominator in the definition (3.19) of $C_{n, d, p}$ is bounded from above by

$$
a_{0}^{-p}\left(a_{0}^{-1}+\frac{1}{p M}\right)^{d} \leq 2 a_{0}^{-(p+d)}
$$


where $n$ is large enough such that

$$
p M \geq \frac{a_{0}}{2^{1 / d}-1} .
$$

This proves $a_{0}^{-(p+d)} / 2<C_{n, d, p}$.

We shall next prove that $\int\|u\|^{p} d G \leq K$. By definition of $G$,

$$
\begin{aligned}
\int\|u\|^{p} d G & =C_{n, d, p} \sum_{u_{1}, \ldots, u_{d} \in S}\left\{\left(u_{1}^{2}+\ldots+u_{d}^{2}\right)^{p / 2}\left(\sum_{j=1}^{d} u_{j}-d a_{0}+a_{0}\right)^{-(p+d)}\right\} \\
& \leq a_{0}^{(d+p)} d^{p} \sum_{u_{1}, \ldots, u_{d} \in S}\left\{u_{1}^{p}\left(\sum_{j=1}^{d} u_{j}-d a_{0}+a_{0}\right)^{-(p+d)}\right\} \\
& =a_{0}^{(d+p)} d^{p} \sum_{u_{1} \in S} u_{1}^{p}\left\{\sum_{\ell_{2}, \ldots, \ell_{d}}\left(a_{0}+\sum_{j=1}^{d}\left(\ell_{j}-1\right) M\right)^{-(p+d)}\right\} \\
& \leq a_{0}^{(d+p)} d^{p} \sum_{u_{1} \in S} u_{1}^{-1} \prod_{j=2}^{d}\left(a_{0}^{-1}+\frac{1}{M(p+j-1)}\right) .
\end{aligned}
$$

It suffices to show $\sum_{u_{1} \in S} u_{1}^{-1}$ is bounded above by a constant. Indeed,

$$
\begin{aligned}
\sum_{u_{1} \in S} u_{1}^{-1} & =\sum_{\ell=1}^{k_{0}}\left(a_{0}+(\ell-1) M \log (\ell e)\right)^{-1} \\
& \leq a_{0}^{-1}+\sum_{\ell=2}^{k_{0}}\left(a_{0}+(\ell-1) M \log (\ell e)\right)^{-1} \\
& \leq a_{0}^{-1}+\sum_{\ell=1}^{k_{0}-1}\left(a_{0}+\ell M \log (\ell e)\right)^{-1} \\
& \leq a_{0}^{-1}+\left(a_{0}+M\right)^{-1}+\int_{1}^{k_{0}}(x M \log x)^{-1} \\
& \leq 2 a_{0}^{-1}+\frac{\log \log \left(k_{0}\right)}{M} \leq 3 a_{0}^{-1}
\end{aligned}
$$

where the last inequality follows by assuming $n$ large enough so that

$$
\frac{\log \log \left(k_{0}\right)}{M} \leq a_{0}^{-1}
$$

This gives

$$
\int\|u\|^{p} d G \leq a_{0}^{(d+p)} d^{p} 3 a_{0}^{-1}\left(a_{0}^{-1}+\frac{1}{M p}\right)^{d-1} \leq 6 d^{p} a_{0}^{p} .
$$


The only remaining thing is to prove (3.21). For convenience, for the index $j^{\prime} \in\{1, \ldots, d\}$, we let $A_{j^{\prime}}=A_{j^{\prime}}\left(\ell_{1}, \ldots, \ell_{j^{\prime}}\right):=a_{0}+\sum_{j=1}^{j^{\prime}}\left(\ell_{j}-1\right) M$. Note that $A_{j^{\prime}} \geq a_{0}$ for all $j^{\prime}=1, \ldots, d$. We need to show that for $j^{\prime} \geq 2$,

$$
\sum_{\ell_{2}}^{k_{0}} \cdots \sum_{\ell_{j^{\prime}}}^{k_{0}} A_{j^{\prime}}^{-\left(p+j^{\prime}\right)} \leq A_{1}^{-(p+1)} \prod_{j=2}^{j^{\prime}}\left(a_{0}^{-1}+\frac{1}{M(p+j-1)}\right)
$$

For the above, we use the idea of mathematical induction on $j^{\prime}$. When $j^{\prime}=2$,

$$
\begin{aligned}
\sum_{\ell_{2}=1}^{k_{0}} A_{2}^{-(p+2)} & =\sum_{\ell_{2}=1}^{k_{0}}\left(a_{0}+\left(\ell_{1}-1\right) M+\left(\ell_{2}-1\right) M\right)^{-(p+2)} \\
& =\sum_{\ell_{2}=1}^{k_{0}}\left(A_{1}+\left(\ell_{2}-1\right) M\right)^{-(p+2)} \\
& \leq A_{1}^{-(p+2)}+\int_{1}^{k_{0}}\left(A_{1}+(x-1) M\right)^{-(p+2)} d x \\
& \leq A_{1}^{-(p+1)}\left(a_{0}^{-1}+\frac{1}{(p+1) M}\right)
\end{aligned}
$$

where the last inequality follows since $A_{1} \geq a_{0}$. Let the above claim (3.22) is true for $j^{\prime}=j_{0}(\geq 2)$. Then, we consider the case $j^{\prime}=j_{0}+1$.

$$
\begin{aligned}
\sum_{\ell_{1}=2}^{k_{0}} \cdots & \sum_{\ell_{j_{0}}=1}^{k_{0}} \sum_{\ell_{j_{0}+1}=1}^{k_{0}} A_{j_{0}+1}^{-\left(p+j_{0}+1\right)}= \\
& \sum_{\ell_{2}=1}^{k_{0}} \cdots \sum_{\ell_{j_{0}}=1}^{k_{0}} \sum_{\ell_{j^{\prime}}=1}^{k_{0}}\left(A_{j_{0}}+\left(\ell_{j^{\prime}}-1\right) M\right)^{-\left(p+j_{0}+1\right)} \\
& =\sum_{\ell_{2}=1}^{k_{0}} \cdots \sum_{\ell_{j_{0}}=1}^{k_{0}}\left(A_{j_{0}}^{-\left(p+j_{0}+1\right)}+\int_{1}^{k_{0}}\left(A_{j_{0}}+(x-1) M\right)^{-\left(p+j_{0}+1\right)} d x\right) \\
& \leq \sum_{\ell_{2}=1}^{k_{0}} \cdots \sum_{\ell_{j_{0}}=1}^{k_{0}}\left\{A_{j_{0}}^{-\left(p+j_{0}\right)}\left(a_{0}^{-1}+\frac{1}{M\left(p+j_{0}\right)}\right)\right\} \\
& \leq A_{1}^{-(p+1)} \prod_{j=2}^{j_{0}}\left(a_{0}^{-1}+\frac{1}{M(p+j-1)}\right)\left(a_{0}^{-1}+\frac{1}{M\left(p+j_{0}\right)}\right) \\
& =A_{1}^{-(p+1)} \prod_{j=2}^{j_{0}+1}\left(a_{0}^{-1}+\frac{1}{M(p+j-1)}\right)
\end{aligned}
$$

where the last inequality follows by the induction hypothesis. 


\section{References}

[1] Doss, N., Wu, Y., Yang, P. and Zhou, H. H. (2020) Optimal estimation of high-dimensional Gaussian mixtures. http://arxiv.org/abs/2002. 05818.

[2] Ghosal, S. and van der Vaart, A. (2001) Entropies and rates of convergence for maximum likelihood and Bayes estimation for mixtures of normal densities. Annals of Statistics, 29, 1233-1263. MR1873329

[3] I. S. Gradshteyn, I. S. and Ryzhik, I. M. Table of Integrals, Series, and Products. Academic Press, 7th edition, 2007. MR0669666

[4] Ho, N. and Nguyen, X. (2016). Convergence rates of parameter estimation for some weakly identifiable finite mixtures. Annals of Statistics, 44, 27262755. MR3576559

[5] Ibragimov, I. (2001). Estimation of analytic functions. In C. Klaasen M. de Gunst and A. W. van der Vaart, editors, State of the Art in Probability and Statistics, IMS, Beachwood, OH., 36, 359-383. MR1836570

[6] Jiang, W. and Zhang, C-H. (2017). Generalized maximum likelihood empirical Bayes estimation of normal means. Annals of Statistics, 37, 1647-1684. MR2533467

[7] Kim, A. K. H. (2014). Minimax bounds for estimation of normal mixtures. Bernoulli, 20, 1802-1818. MR3263090

[8] Li, J. and Schmidt, L. (2017). Robust and proper learning for mixtures of Gaussians via systems of polynomial inequalities. Conference on Learning Theory, 1302-1382. MR4262509

[9] Polyanskiy, Y. and Wu, Y. (2021). Sharp regret bounds for empirical Bayes and compound decision problems. https://arxiv.org/pdf/2109.03943. pdf.

[10] Rahman, S. (2017). Wiener-Hermite polynomial expansion for multivariate Gaussian probability measures. Journal of Mathematical Analysis and Applications, 454, 303-334. MR3649855

[11] Saha, S. and Guntuboyina, A. (2017). On the nonparametric maximum likelihood estimator for Gaussian location mixtures densities with application to Gaussian denoising. Annals of Statistics, 48, 738-762. MR4102674

[12] Suresh, A. T., Orlitsky, A., Acharya, J., and Jafarpour, A. (2014). Nearoptimal-sample estimators for spherical Gaussian mixtures. Advances in Neural Information Processing Systems, 1395-1403.

[13] Van der Vaart, A. (2000). Asymptotic statistics. Cambridge university press, Cambridge, United Kingdom, 2000. MR1652247

[14] $\mathrm{Wu}, \mathrm{Y}$. and Yang, P. (2020). Optimal estimation of Gaussian mixtures via denoising method of moments. Annals of Statistics, 48, 1981-2007. MR4134783

[15] Zhang, C-H. (2009). Generalized maximum likelihood estimation of normal mixture densities. Statistica Sinica, 19, 1297-1318. MR2536157 